\title{
Modelling of routing and spectrum handoff in CR-MANETs
}

\author{
Samad Nejatian ${ }^{1,2^{*}}$, Sharifah Kamilah Syed-Yusof', Nurul Muazzah Abdul Latiff' and Wajahat Maqbool'
}

\begin{abstract}
There is an extensive research interest in cognitive radio mobile ad hoc networks (CR-MANETs) to improve the spectrum efficiency by developing innovative design techniques through various layers of the protocol stacks. This paper presents the optimisation of CR-MANETs by exploiting the efficient usage of the available wireless spectrum through a framework for spectrum-aware handoff. In this paper, the concept of integrated handoff management in CR-MANETs is considered. An analytical model for a spectrum handoff scheme is introduced based on spectrum mobility in which secondary users (SUs) will move to another unused spectrum band, giving priority to a Primary User (PU), while satisfying its communication quality of service (QoS). The main contribution is using the Markov chain to model the evolution of the network (node position, node speed, channel quality, etc.) and to propose the combined spectrum handoff and routing. The performance of the network is analyzed based on the Markov chain. The comparison results from both analytical modelling and simulation clearly show an improvement in the performance of the SU network in terms of the route maintenance probability and the SU throughput. It is also proved that not only the PU activity affects the performance of the handoff management scheme but also the channel transmission range and the node mobility have a significant effect on the performance of the management scheme.
\end{abstract}

Keywords: Cognitive radio; Mobile Ad hoc networks; Spectrum handoff; Spectrum mobility

\section{Introduction}

Recently, there have been major changes in wireless technologies that demand more spectrum bands for allocation to emerging wireless applications. The Federal Communication Commission (FCC) has shown low spectrum efficiency usage in current wireless networks. Low and poor spectrum efficiency is due to the underutilized spectrum usage because of the fixed spectrum allocation [1]. The most influential technology that promises to improve spectrum efficiency is cognitive radio (CR) [2]. CR users can capture and use the unused spectrum bands called spectrum holes [3].

The spectrum holes may shift over time and over space $[4,5]$. In the CR system, the shifting of the spectrum holes is defined as spectrum mobility, which is cohesive with spectrum handoff. Spectrum handoff refers to the transfer of the ongoing data transmission of a CR user to another available spectrum band [6,7]. During the spectrum handoff, a CR user will move to another unused spectrum band,

\footnotetext{
* Correspondence: samad.nejatian.ir@ieee.org

'UTM-MIMOS Center of Excellence, Faculty of Electrical Engineering, Universiti Teknologi Malaysia (UTM), Johor Bahru 81310, Malaysia

${ }^{2}$ Department of Electrical Engineering, Faculty of Engineering, Yasouj Branch, Islamic Azad University, Yasouj, Kohgiloyeh \& Bovirahmad Province, Iran
}

giving priority to a Primary User (PU), while satisfying its communication quality of service (QoS) $[8,9]$. Spectrum handoff is extremely challenging in CR networks, especially in cognitive radio mobile ad hoc networks (CR-MANETs), because of frequent topology variations, limited power and channel transmission range and bandwidth constraints in addition to a lack of a central controlling entity. In heterogeneous CR networks, a channel may be available over vast mutually exclusive spectrum bands, which present remarkable heterogeneity in terms of channel transmission range and channel error rate.

The challenges in maintaining a common link arise when the secondary user (SU) nodes move. The first challenge is differentiating the SU's mobility and spectrum mobility. Channel quality degradation happens because of the SU's mobility and channel heterogeneity in terms of transmission range. Therefore, there are different route failure types that necessitate different route recovery strategies. The second challenge related to integrated handoff management is finding the best new route and a channel to maintain the route while reducing the switching time and the number of handoffs. In multi-hop CRNs, routing 
is a crucial issue which affects the performance of the whole network [10]. When considering the influence of node mobility on the channel availability, the effect of channel heterogeneity becomes more significant. In a CR system, node mobility and channel heterogeneity lead to frequent spectrum handoffs [11].

Previous work on spectrum handoff in CR networks only considers the PU's activity. In this paper, we consider the integrated handoff management scheme for CR-MANETs. The work considers the CR spectrum handoff problem and jointly addresses the local flow handoff issue. To propose the integrated handoff management scheme, the availability of spectrum holes in CR-MANETs is considered. The influence of different parameters and events on channel availability is considered in the analytical scheme to obtain a unified model for channel availability in CRMANETs. The Markov chains are used to model the integrated handoff process in CR-MANETs. The effect of the integrated handoff management on the improvement of the handoff blocking probability is also demonstrated.

The rest of this paper is organized as follows: Section 2 describes the related works for spectrum handoff management. In Section 3, we propose a model for integrated spectrum handoff management and routing scheme. In Section 4, we propose a unified modelling and characterization of the channel availability in CR-MANETs. Section 5 shows the analytical model of the integrated spectrum handoff management. In Section 6, the results and discussion are elaborated. Finally, Section 7 concludes the paper and presents future work.

\section{Related works}

There are a few studies related to spectrum handoff in CRMANETs. Giupponi and Perez-Neira [12] proposed a fuzzy-based spectrum handoff decision-making approach employing two fuzzy logic controllers. Each SU estimates the distances between itself and all the active PUs in the surrounding area using the first fuzzy logic controller. The other fuzzy logic controller determines whether the SU needs to perform a spectrum handoff. In some cases, the SU can avoid performing a spectrum handoff by appropriate adjustment of its transmission power. Feng et al. developed a spectrum handoff technique from a single-link concept to a multi-link spectrum handoff scheme [13]. The proposed algorithm attempts to minimize the total link cost by taking into account the end-to-end network connectivity constraint. Another major contribution of this paper was that the rerouting mechanism was performed before a spectrum handoff event to increase the system throughput. Song and Xie [14,15] proposed a proactive spectrum handoff configuration based on statistics of observed channel utilization. The network coordination and rendezvous issues were solved in this spectrum handoff scheme without using a common control channel. The collision among SUs was prevented through a distributed channel determination scheme. Damljanovic explained the proper solutions and spectrum mobility necessities in cognitive radio networks [16]. Duan and Li proposed a spectrum handoff strategy in which the optimal spectrum band was chosen based on a multiplex criterion considering the estimated transmission time, the PU presence probability and the spectrum availability time [17]. A cooperative spectrum sensing scheme was used to predict the spectrum idleness. A geo-location method was used to perform a spectrum handoff in the space domain. The simulation results indicated that the proposed spectrum handoff scheme outperformed conventional methods in terms of spectrum handoff delay in a per hop basis. However, channel heterogeneity parameters are not considered in the spectrum handoff. Wu and Harms [18] proposed a proactive flow handoff for legacy mobile ad hoc networks. The major contribution of this scheme was maintaining end-to-end connectivity after a flow was established. This scheme introduced the consideration of user mobility and location information. Abhilash et al. [19] proposed a preemptive route maintenance scheme in which an established route is repaired before it breaks by considering the mobile ad hoc user's location information. They called this scheme local router handoff and implemented it into the ad hoc on-demand distance vector protocol (AODV). Based on the results, the throughput of the system was increased under certain conditions. A novel channel allocation scheme for SUs is proposed in [20]. In this study, an analytical model is proposed in which the Markov models are used to model the behaviour of both PU and SU. In this research, the on/off model is combined with the traditional queuing analysis model to show the effectiveness of the proposed method on the SU's channel allocation. Chehata et al. [21] introduced the CR-AODV as a multi-radio, multi-channel, on-demand scheme that can manage the data transmission of cognitive users. Cacciapuoti et al. [22] proposed a reactive routing protocol by evaluating the feasibility of reactive routing for CR-MANETs. In [23], the integration of an energy-saving routing scheme and the open shortest path first (OSPF) protocol is used. The proposed integrated scheme allows the selection of the links to be switched off so that the negative effects of the topology reconfiguration processes are avoided. Handoff management in CR-MANETs was pioneered in [24]. Factors and types of mobility were mentioned, which necessitate integrated mobility and handoff management in CR-MANETs. In this paper, a conceptual model was proposed for integrated handoff management in CR-MANET. Nejatian et al. [25] characterized and formulated the availability of spectrum bands in CR-MANETs. They explained and integrated the 
effects of various events on the availability of spectrum holes in CR-MANETs [26]. In [27], Nejatian et al. introduced a new algorithm for integrated handoff management in which the handoff is performed considering the effects of all the parameters introduced in [25]. The study reveals that the channel heterogeneity and SU's mobility must be considered as important factors, which affect the performance of the handoff management in the CR-MANETs.

As mentioned before, maintaining the optimal routes in CR-MANETs is extremely difficult because of the randomness of the PU's activity, the SU's mobility and the channel quality degradation. Although $[27,28]$ introduced and investigated the integrated handoff management in CRMANETs as a new algorithm, analytical modelling of this concept can be also efficient. Hence, a framework is introduced to analytically model the integrated handoff management in CR-MANETs. The results of both analytical modelling and proposed algorithm are compared to show the effectiveness of the proposed solution.

\section{Spectrum hole availability in CR-MANETs 3.1 System description}

Suppose there are $L$ different channel types in a heterogeneous PU network. The maximum number of channels that can be accessed by the $\mathrm{SU}$ at a time is $C$. The $C$ channels can be defined by the set of $\mathbf{T}$, which belong to the PU network. These channels are classified into $L$ types according to their different transmission ranges that the cardinality of $|\mathbf{T}|=L$ (see Figure 1). Channels with different transmission ranges belong to a different set of spectrum pools. The set of each type can be shown by $T_{l}$ in which $l \in \mathbf{S}, \mathbf{S}=\{1,2, \ldots, L\},\left|T_{l}\right|=C_{l}, \mathbf{T}=\left\{T_{1}, \ldots, T_{L}\right\}$ and $C=C_{1}+\ldots+C_{L}$. Depending on the PU activities, any SU can access up to $C$ channels at any position. The number of detected channels of type $l$ by a special node is $c_{l}$, and the total number of detected channels at a node is $c=c_{1}+$ $c_{2}+\ldots+c_{L}$. The transmission range of channels of type $T_{l}$ is $R_{l}$. Given the channel transmission range classification, we can model the channel heterogeneity. Assume a pair of SU transmitter-receivers, which transmit and receive, respectively, using channel of type $T_{l}$ for communication, with a distance between them of less than $R_{l}$. When the SUs move and their distance exceeds $R_{l}$, the transmitting node must change and choose another channel. In this case, the required channel must have a transmission range longer than $R_{l}$ (Figure 2). There are also $L$ types of PU; each of them can work only on a channel of type $l$. Once a PU of type $l$ becomes active and there is no empty channel of type $l$, in the case which a SU has occupied a channel of type $l$, the SU must vacate the channel and submit it to the PU.

\subsection{Characterization of channel availability and spectrum mobility in CR-MANETs}

First, only the effect of the PU activity is considered on the channel availability in CR-MANETs. Table 1 shows the different parameters and their definitions used in this paper.

Consider an established route from the source node $\mathrm{S}$ to the destination node $\mathrm{D}$. We define the parameter $p$ as the probability of single channel availability at a node by considering the PU activity, and $P_{\text {cat }}$ is the probability of single channel availability per hop between two nodes. Because the channel availability on all nodes is independent of each other, we can consider different hops separately to estimate the probability of channel availability through the established route. Thus, $P_{\text {cat }}$ is as follows:

$$
P_{\text {cat }}=p^{2}
$$

Consequently,

$$
\bar{P}_{\text {cat }}=1-p^{2}
$$

The effects of the spectrum heterogeneity and mobility of the SU on the probability of channel availability is considered. In a heterogeneous network, each channel experiences various levels of packet error rate (PER) and different

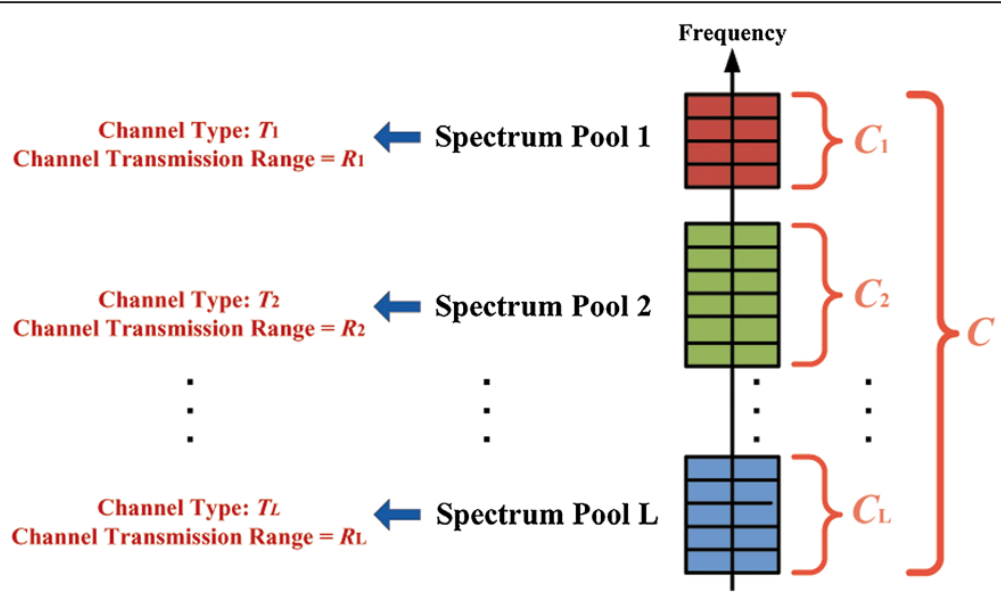

Figure 1 Spectrum-pool-based heterogeneous CR-MANET scheme. 


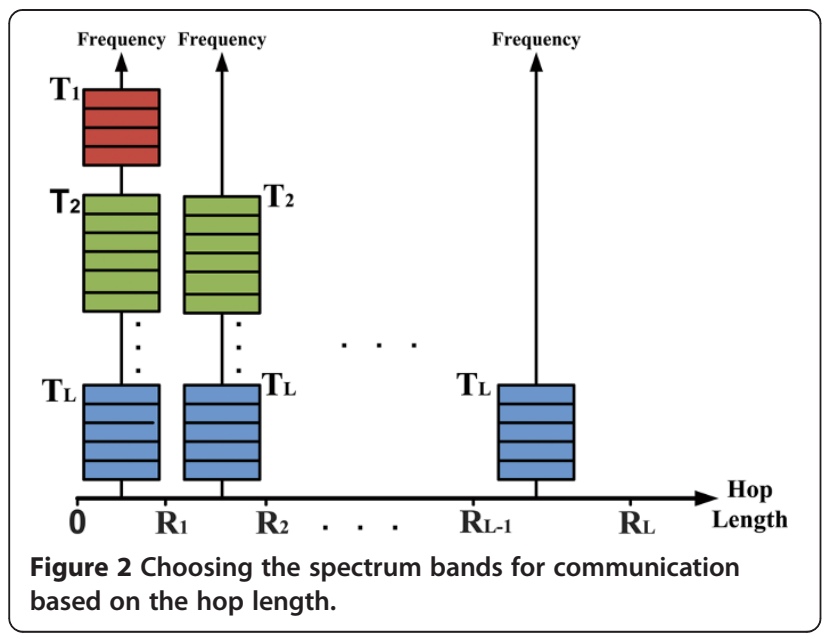

channel transmission ranges. In the initial condition when the nodes are assumed to be fixed, different channels will have different transmission ranges. A channel with a lower frequency range needs lower transmission power. Thus, in a heterogeneous network with different channel transmission ranges, the distance between the SUs affects the probability of channel availability.

We define $\bar{P}_{\text {cat }, c_{l}}$ as the probability of unavailability of a single channel of type $l$ among $c_{l}$ channels between two nodes as follows:

$$
\bar{P}_{\text {cat }, c_{l}}=\left(\bar{P}_{\text {cat }}\right)^{c_{l}}=\left(1-p^{2}\right)^{c_{l}}
$$

Based on (3), the probability that there is at least one single channel of type $T_{l}$ among $c_{l}$ channels between two nodes is

$$
P_{\text {cat }, c_{l}}=1-\bar{P}_{\text {cat }, c_{l}}=1-\left(1-p^{2}\right)^{c_{l}}
$$

\section{Table 1 The different parameters used and their definitions}

\begin{tabular}{ll}
\hline Symbol & Definition \\
\hline$R_{T}$ & Transmission range \\
$C$ & Total number of available channels \\
$l$ & Number of detected channels at each node \\
$L$ & Channel type \\
$C_{l}$ & Total number of channel types \\
$N$ & Number of possible channels of each type at each node \\
$N_{N}$ & Number of nodes in a route \\
$A_{N}$ & Total number of the nodes in the network \\
$P$ & Network area \\
$\lambda$ & Probability of a particular channel availability at each node \\
$R_{l}$ & Transmission range of a channel of type I \\
\hline
\end{tabular}

The probability of channel availability within a hop depends on the distance between nodes, $d$. The $P\left(R_{i}\right)$ is defined as the probability that the length of the hop between two adjacent nodes in the route is less than $R_{i}$ and longer than $R_{i}-1$. The distances between adjacent nodes in a route are independent random variables. Therefore considering (4) and the law of the total probability, it can be concluded that:

$$
\begin{aligned}
P_{\text {cat }, c}= & P\left(R_{1}\right)\left[1-\left(1-p^{2}\right)^{c}\right]+P\left(R_{2}\right)\left[1-\left(1-p^{2}\right)^{c-c_{1}}\right]+\ldots \\
+ & P\left(R_{L}\right)\left[1-\left(1-p^{2}\right)^{c_{L}}\right]=\sum_{i=1}^{L} P\left(R_{i}\right)\left[1-\left(1-p^{2}\right)^{c-} \sum_{j=0}^{i-1} c_{j}\right] \\
& \text { in which } c_{0}=0, R_{0}=0, i=1,2, \ldots, L .
\end{aligned}
$$

Consequently, based on (4) and (5), and the existence of $n-1$ hops through a route, we have

$$
P_{\mathrm{car}, c}=\left(P_{\mathrm{cat}, c}\right)^{n-1}
$$

Consequently, based on (5) and (6), we have

$$
P_{\mathrm{car}, c}=\left[\sum_{i=1}^{L} P\left(R_{i}\right)\left[1-\left(1-p^{2}\right)^{c-} \sum_{j=0}^{i-1} c_{j}\right]\right]^{n-1}
$$

Let the node transmission power be fixed to $R_{T}$. In fact, the transmitting range with a similar transmitting power on wireless spectrum bands with different frequencies is unequal. We assume that $R_{1}<\ldots<R_{l}<R_{T}$. The probability of $P\left(R_{i}\right)$ is dependent on the number of hops and also the limit of the transmission range. To demonstrate the effects of channels with different transmission ranges on the system, we model the spatial distribution of mobile nodes as a Poisson variable with a density $\lambda$. Thus [29],

$$
\begin{aligned}
& \operatorname{Pr}(\text { There are } k \text { nodes in a region with disc area } S) \\
& \quad=\frac{\lambda S^{k} \exp (-\lambda S)}{k !}, k=0,1,2,3, \ldots
\end{aligned}
$$

Without the loss of generality, this can be considered as a snapshot of the mobile radio network, by which we can calculate $P\left(R_{i}\right)$. The number of neighbouring nodes in a disc area with radius $R_{T}$ is equal to

$$
N=\lambda \pi R_{T}^{2}
$$


The probability that there is no neighbour node in the forward direction in the disc with radius $R_{T}$ is also expressed as $\exp \left(-^{N} / 2\right)$; therefore,

$$
\left.P\right|_{\text {finding a node in a disc with radius } R_{T}}=1-\exp (-N / 2)
$$

Assume that the random variable $d$ denotes the distance between a pair of transmitter and receiver nodes and the $f_{d}\left(R_{i}\right)$ represents its probability density function (pdf). The probability distribution function of $d$ is as follows:

$$
\begin{aligned}
F_{d}\left(R_{i}\right) & =\operatorname{Pr}\left(d<R_{i}\right) \\
& =\frac{1-\exp \left(-\frac{\lambda \pi R_{i}^{2}}{2}\right)}{1-\exp \left(-\frac{N}{2}\right)} \quad, 0<R_{i}<R_{T}
\end{aligned}
$$

Thus, we can conclude that

$$
\begin{aligned}
\operatorname{Pr}\left(R_{i-1}<d<R_{i}\right)= & F_{d}\left(R_{i}\right)-F_{d}\left(R_{i-1}\right) \\
= & \frac{1-\exp \left(-\lambda \pi R_{i}{ }^{2} / 2\right)}{1-e^{-\frac{N}{2}}} \\
& -\frac{1-\exp \left(-\lambda \pi R_{i-1}{ }^{2} / 2\right)}{N} \\
= & \frac{\exp \left(-\lambda \pi R_{i-1}{ }^{-\frac{N}{2}} / 2\right)-\exp \left(-\lambda \pi R_{i}{ }^{2} / 2\right)}{1-e^{-\frac{N}{2}}}
\end{aligned}
$$

in which $0<R_{i-1}<R_{i}<R_{T} \quad R_{0}=0$

Finally, we have

$$
P_{\mathrm{car}, c}=\left[\sum_{i=1}^{L} \frac{\exp \left(-\lambda \pi R_{i-1}^{2} / 2\right)-\exp \left(-\lambda \pi R_{i}^{2} / 2\right)}{1-e^{-\frac{N}{2}}}\left[1-\left(1-p^{2}\right)^{c-\sum_{j=0}^{i-1} c_{j}}\right]\right]^{n-1}
$$

Based on the channel availability modelling, the unified spectrum handoff scheme must be proposed to include different mobility events in CR-MANETs, such as spectrum and user mobility, channel quality degradation and topologic variation.

\section{Routing and integrated spectrum handoff management in CR-MANETs}

\subsection{Proposed integrated framework}

Figure 3 shows the framework for integrated spectrum handoff management and routing in CR-MANETs. The proposed framework considers the spectrum-aware handoff management based on interactions between routing (layer 3) and the physical layer (layer 1). The proposed scheme is equipped with an algorithm to identify appropriate spectrum bands based on the channel qualities, the spectrum and the node mobility. The spectrum analysis identifies information about the situations and the mobility of the SUs. Thus, the existence of a precise and cooperative environment and a locationaware mechanism is necessary.

In the next subsections, the analytical modelling of the routing and integrated handoff management in CR-MANETs is performed considering the spectrum mobility and channel availability characterized in the previous section.

\subsection{Analytical modelling of routing}

A wireless network of $m$ nodes is modelled as a directed graph $G(V, E)$ [30]. As Figure 4 shows, consider a path (SD) from source node $\mathrm{S}$ to destination node $\mathrm{D}$ consisting of a sequence of $n$ nodes and consequently $n-1$ undirected hops. The states active (A) and unactive (U) are defined for the different hops and the nodes of the rout considering their condition. A node in the route is in the state A at time $t$ when there is at least one available channel on this node. A node is in the state $U$ when there is no available channel on this node. A link in the route is in the state A when its end point nodes are within transmission range of each other independently of the states in which they are; otherwise, the link is said to be in the state U. A hop is said to be active when its two end point nodes are in the A state while they are within transmission range of each other (the link is in the A state) and they are in active state. A route exists when all its hops are in state A. Suppose $s_{v}=\left\{n_{1}=S, n_{2}, \ldots, n_{i}, \ldots, n_{n}=D\right\}$ be the sequence of $n$ nodes and $s_{e}=\left\{e_{1}, e_{2}, \ldots, e_{i}, \ldots, e_{n-1}\right\}$ be the sequence of $n-1$ links composing path $(S D)$ with $n-1$ hops.

Consider link $e_{i}$ with the $n_{i}$ and $n_{i+1}$ as its end nodes, the probability of being in active state of $i_{t h}$ hop $\left(h_{i}\right)$ at time $t$ is as follows:

$$
P_{\mathrm{A}}\left(h_{i}, t\right)=P_{\mathrm{A}}\left(n_{i}, t\right) \cdot P_{\mathrm{A}}\left(n_{i+1}, t\right) \cdot P_{\mathrm{A}}\left(e_{i}, t\right)
$$

where $P_{\mathrm{A}}\left(n_{i}, t\right)$ is the probability that node $n_{i}$ is in state A at time $t$ and $P_{\mathrm{A}}\left(e_{i}, t\right)$ is the probability that nodes $n_{i}$ and $n_{i+1}$ are within transmission range of each other at time $t$. The probability of existence of the route from a source node $\mathrm{S}$ to a destination node $\mathrm{D}$ at time $t$ is as follows:

$$
P_{\mathrm{E}}\left(u_{\mathrm{SD}}\right)=\prod_{n_{i} \in S_{\nu}} P_{\mathrm{A}}\left(n_{i}, t\right) \prod_{e_{i} \in S_{e}} P_{\mathrm{A}}\left(e_{i}, t\right)
$$

The objective is to find the optimal route between the source and destination which is the one with the greatest probability of existence among all possible paths. Suppose 


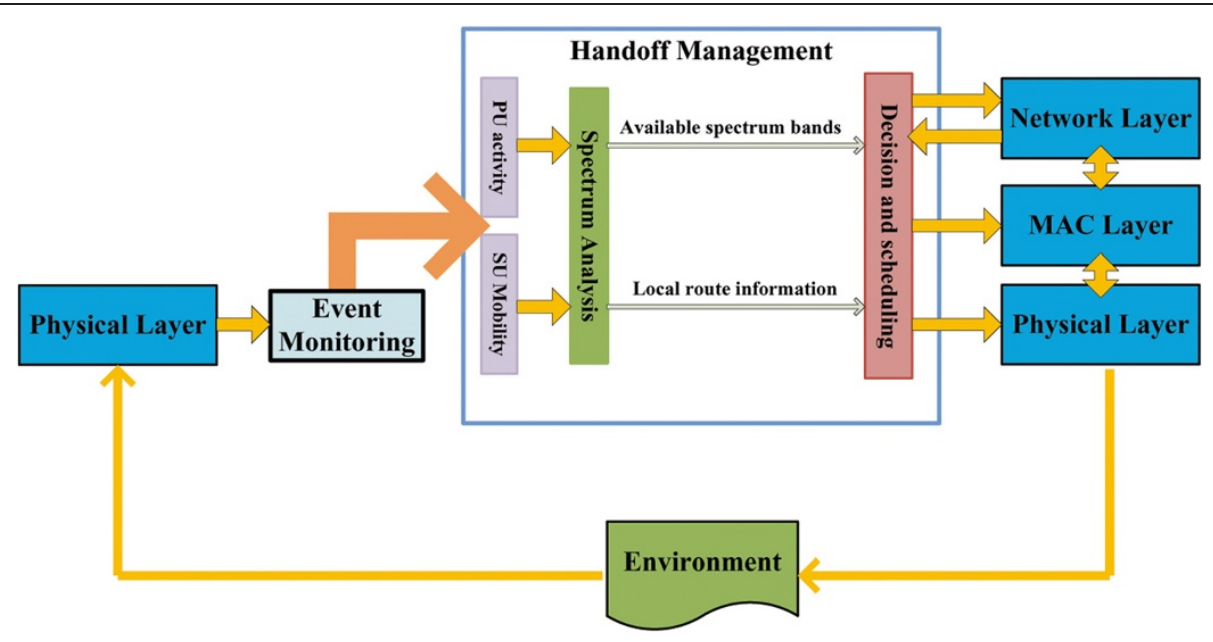

Figure 3 Functional block diagram for integrated mobility and handoff management.

that the $U_{(\mathrm{SD})}$ be the set of all possible routes from source node $\mathrm{S}$ to destination node $\mathrm{D}$. The $u_{\mathrm{SD} \text {,opt }}$ which is the optimum route from source node $\mathrm{S}$ to destination node $\mathrm{D}$ with the greatest existence probability is then given by

$$
u_{\mathrm{SD}, \mathrm{opt}}: \operatorname{Arg} \max _{u_{\mathrm{SD}} \in U_{(\mathrm{SD})}} P_{\mathrm{E}}\left(u_{\mathrm{SD}}\right)
$$

Consequently

$$
u_{\mathrm{SD}, \mathrm{opt}}: \operatorname{Arg} \max _{u \in U_{(\mathrm{SD})}} \ln P_{\mathrm{E}}\left(u_{\mathrm{SD}}\right)
$$

And it can be concluded that

$$
u_{\mathrm{SD}, \mathrm{opt}}: \operatorname{Arg} \max _{u_{\mathrm{SD}} \in U_{(S D)}}\left(\ln \left(\prod_{n_{i} \in u_{\mathrm{SD}}} P_{\mathrm{A}}\left(n_{i}, t\right) \prod_{e_{i} \in u_{\mathrm{SD}}} P_{\mathrm{A}}\left(e_{i}, t\right)\right)\right)
$$

Considering the unified Equation 13, it can be concluded that

$$
u_{\mathrm{SD}, \mathrm{opt}}: \operatorname{Arg} \max _{u_{\mathrm{SD}} \in U_{(S D)}}\left(\ln \left(P_{\mathrm{car}, c}\left(u_{\mathrm{SD}}\right)\right)\right)
$$

\subsection{Analytical modelling of integrated handoff}

In this part, we exploit Markov chains to model the spectrum handoff mechanism in CR-MANETs based on the channel availability modelling above. We also consider the spectrum heterogeneity in terms of PER. Once the channel quality declines or PER increases, the probability of successful packet transmission rate is decreased. The SU detects this deterioration throughput QoS and decides to change the channel to achieve a better throughput performance. Considering $P_{\mathrm{E}}^{i}$ as the PER of channel of type $i$ and Equation 13, the probability of successful packet routing in a route or between $n$ nodes is deduced as

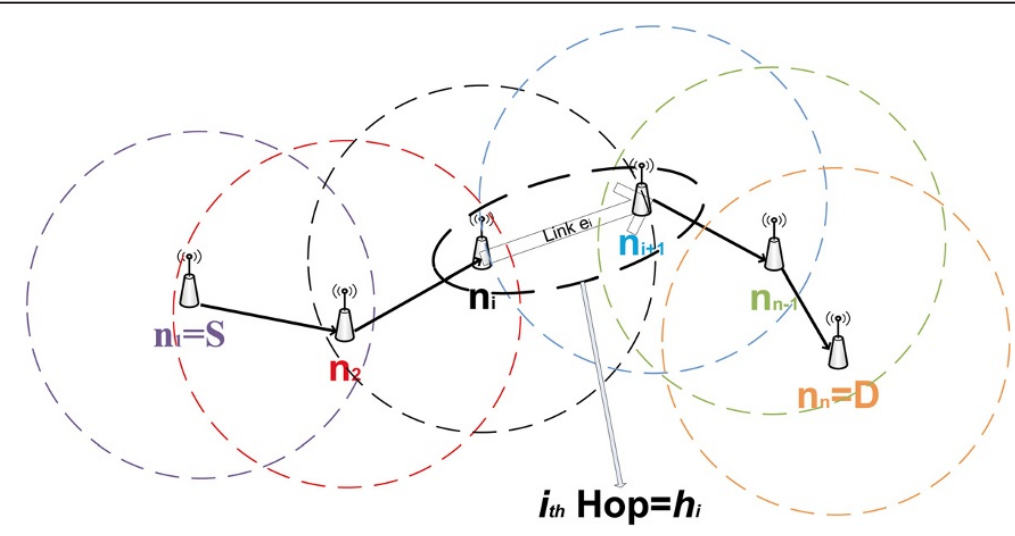

Figure 4 A path (SD) from source node $S$ to destination node $D$. 


$$
p_{\mathrm{spr}, c}=\sum_{i=1}^{L} P_{\mathrm{car}, c}\left(1-P_{\mathrm{E}}{ }^{i}\right)=\left[\sum_{i=1}^{L}\left(1-P_{\mathrm{E}}{ }^{i}\right)\left[\frac{\exp \left(-\lambda \pi R^{2}{ }_{i-1} / 2\right)-\exp \left(-\lambda \pi R^{2} / 2\right)}{1-e^{-\frac{N}{2}}}\right]\left[1-\left(1-p^{2}\right)^{c-} \sum_{j=0}^{i-1} c_{j}\right]\right]^{n-1}
$$

The probability of successful packet transmission in a hop or between two nodes is also found as

$$
p_{\mathrm{spt}, c}=\left.p_{\mathrm{spr}, c}\right|_{n=2}
$$

The parameter $P_{\mathrm{E}}^{i}$ shows the rate of failed packets sent because of the variable channel conditions caused by factors such as fading and shadowing. The probability of unsuccessful packet transmission in a hop or between two nodes is also found:

$$
p_{\text {uspt }, c}=1-p_{\text {spt }, c}
$$

To calculate the probability distribution of spectrum handoff and also model the spectrum handoff initiation in CR-MANETs, we define the position case $D_{l}-1 l$ as the case in which $R_{l}-1<d<R_{l}$, where $d$ is the length of the hop. Hereafter, for the sake of indexing convenience, we refer to the position case $D_{l-1 l}$ as $D_{l}$ throughout the paper. Figure 5 shows the different Markov chains for spectrum handoff modelling based on the length of the hops. P, which is the Markov matrix, is written as follows:

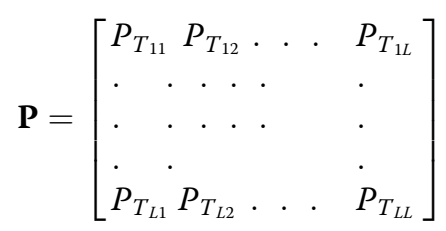

The Markov chains have different numbers of states based on the different position cases $D_{l}$. As shown in Figure 2 previously, when the length of the hop is less than $R_{1}$, or when the spectrum handoff occurs in the position case $D_{1}$, the two nodes involved in the current hop can select one of the available channels of any $L$ types. In the position case $D_{1}$, the Markov chain is as shown in Figure $5 \mathrm{a}$. In the case $D_{2}$, the nodes involved in spectrum handoff can select one channel among available channels from type $k$, in which $k \neq 1$, as shown in Figure $5 \mathrm{~b}$. When the distance between the involved nodes in the spectrum handoff is according to position case $D_{\mathrm{L}}$, the nodes can only select one channel among available channels of type $\mathrm{L}$, as shown in
Figure 5c. The other situations can be determined based on the claims above.

Suppose that two nodes are communicating in a channel of type $k$. There are two different conditions where nodes continue their communication in the current spectrum pool. These two conditions, in the position case of $D_{l}$, are as follows:

- The packet transmission is successful in the current channel of type $k$.

- The packet transmission is not successful in the current channel of type $k$ but only successful on another channel of spectrum pool $k$.

Thus, the probability that the involved nodes in this hop do not switch their channel type, $P_{T_{k k}}$, is calculated as follows:

$$
P_{T_{k k}, D_{l}}=p_{\mathrm{spt}, c_{k}}+\prod_{j=1, j \neq k}^{L} p_{\mathrm{uspt}, c_{j}}
$$

There are also two conditions where two nodes, which are communicating on a channel of type $e$, switch their channel type to another channel of type $k$ such that $k \in$ $\{1,2, \ldots, L\}, k \neq e$. These two conditions, in the position case $D_{l}$, are as follows:

- Unsuccessful packet transmission in a channel of type $e$, but only successful transmission in channel of type $k$.

- Unsuccessful packet transmission in a channel of type $e$, but successful transmission in channel type sets:

$$
\mathbf{M}_{i} \subseteq \mathbf{T},\left|\mathbf{M}_{i}\right|=L_{i}<L
$$

Based on the Markov chains, there are many possible channel type sets for $\mathbf{M}_{i}$. The channel of type $j$ can be chosen with an identical probability among the available channel types in the set of $\mathbf{M}_{i}$. Based on the explanation above, the probability that the involved nodes in this hop switch their channel type is as follows: 


$$
P_{T_{e k}, D_{l}}=\left[p_{\text {uspt }, c_{e}} \times\left(p_{\text {spt }, c_{k}}\left(\prod_{j=1, j \neq e, k}^{L} p_{\text {uspt }, c_{j}}\right)\right)\right]+\left[p_{\text {uspt }, c_{e}} \sum_{\mathbf{M}_{i} \in \mathbf{T}} \frac{\prod_{j=1,2, \ldots, i, T_{j} \in \mathbf{M}_{i}, j \neq e} p_{{\text {sup }, c_{j}}_{j=1,2, \ldots, i, T_{j} \notin \mathbf{M}_{i}, j \neq e}} p_{\text {uspt }, c_{j}}}{\left|\mathbf{M}_{i}\right|}\right]
$$

The row vector $\pi^{\mathbf{P}} D_{l}$, which is composed of $\pi^{\mathrm{P}}{ }_{D_{l}}\left(T_{i}\right)$, demonstrates the steady state probability for $\mathbf{P}_{D_{l}}$ considering different hop lengths. The value of $\pi^{\mathrm{P}}{ }_{D_{l}}\left(T_{i}\right)$ is calculated using the following equations:

$$
\pi^{\mathbf{P}_{D l}} \mathbf{P}_{D_{l}}=\pi^{\mathbf{P}}{ }_{D l}, \sum_{i=1}^{L} \pi_{D_{l}}^{\mathrm{P}}\left(T_{i}\right)=1
$$

Ultimately, the steady state probabilities for various hop lengths for channel type $i$ are calculated as below:

$$
\pi^{\mathrm{P}}\left(T_{i}\right)=\sum_{l=1}^{L} \operatorname{Pr}\left(R_{l-1}<d<R_{l}\right) \pi_{D_{l}}^{\mathrm{P}}\left(T_{i}\right)
$$

where $\operatorname{Pr}\left(R_{l-1}<d<R_{l}\right)$ is calculated using (12).

\subsection{Integrated mobility and spectrum handoff management}

To propose the integrated spectrum handoff management, we introduce the different scenarios which cause spectrum handoff initiation through an established route in a CR-MANET. Suppose that, based on Figure 6, a route from a source node $S$ to the destination node $\mathrm{D}$ has been established. There are three different scenarios that initiate the spectrum handoff in this route, which are as follows:

\subsubsection{SU mobility}

As shown in Figure 6a, route failure occurs when either node $\mathrm{B}$ or node $\mathrm{F}$ moves such that no channel can support their transmission. Before the route failure occurs, local flow handoff is performed. A local flow handoff can be from node $\mathrm{B}$ to node $\mathrm{E}$ and finally joining node $\mathrm{F}$. In this scenario, the unified routing and spectrum handoff
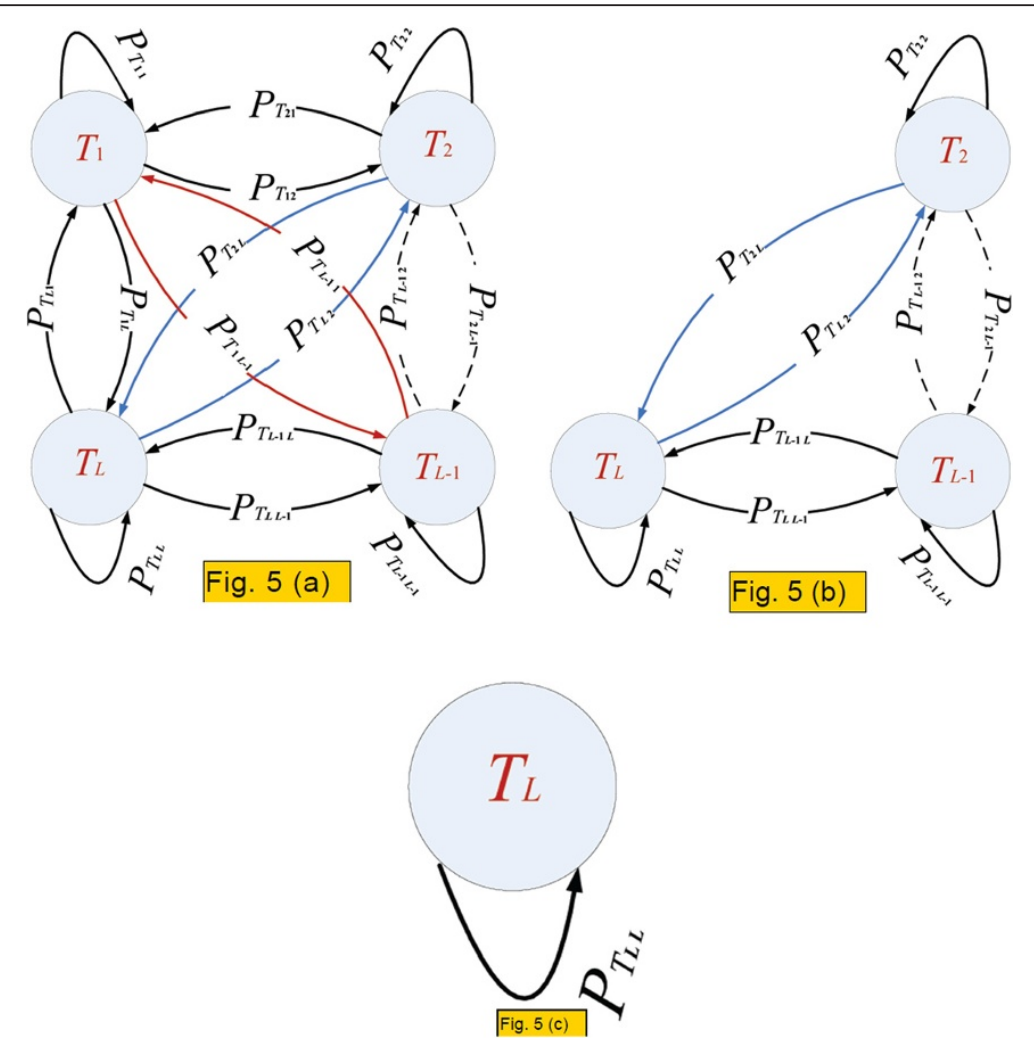

Figure 5 Markov chains for spectrum handoff modelling based on the distance between SUs or the length of the hops. (a) In case $D_{01}$, (b) in case $D_{12}$ and $(\mathbf{c})$ in case $D_{L-1 L}$. 


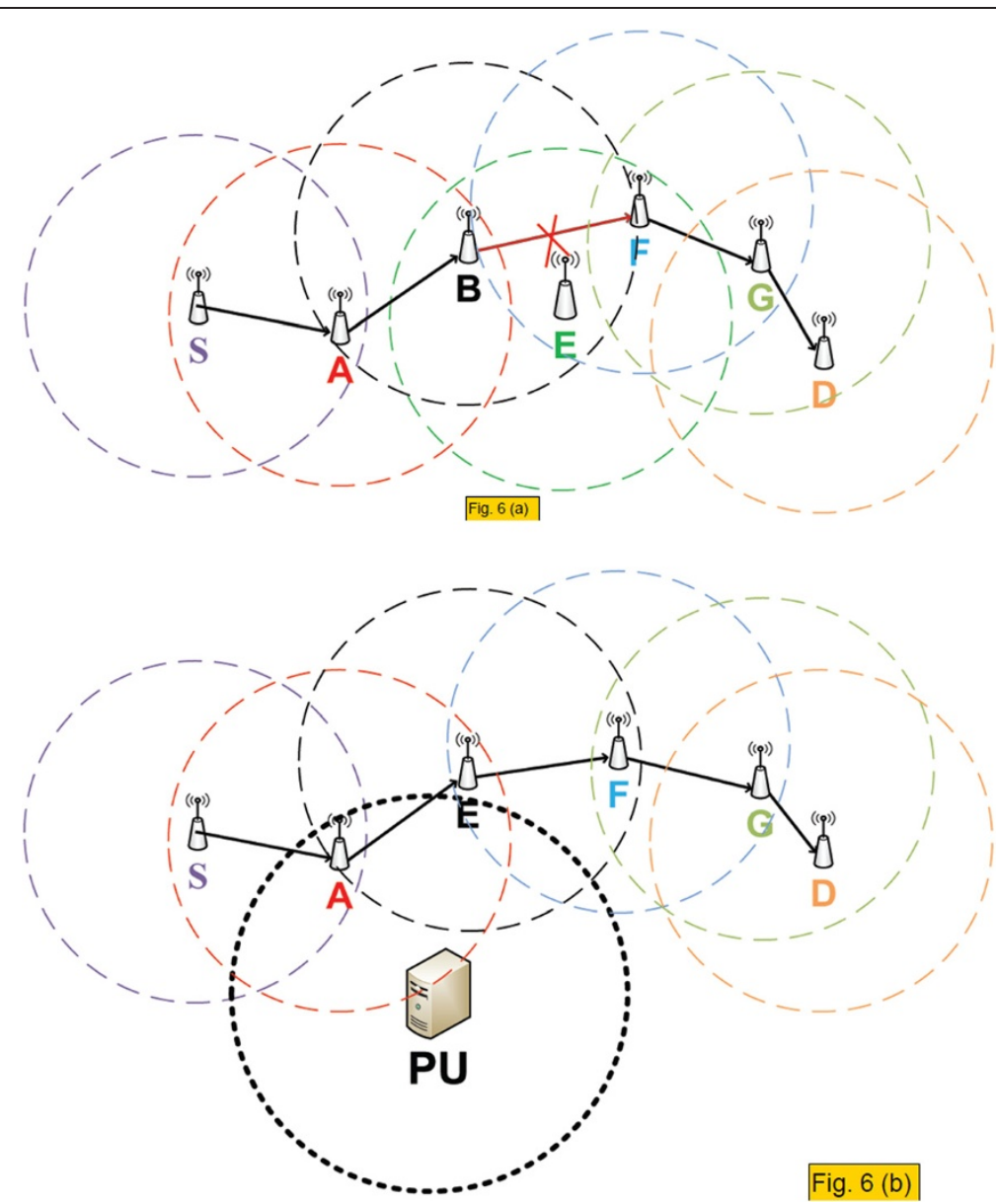

Figure 6 Different scenarios causing spectrum handoff initiation through an established route in CR-MANETs. (a) SU mobility and spectrum heterogeneity and (b) PU activity.

management system tries to solve the problem by finding a node within the neighbouring area of the damaged links.

To perform local routing, a certain amount of overlapping of the transmission range between node $B$, node $F$ and the node that will take responsibility for routing the packets

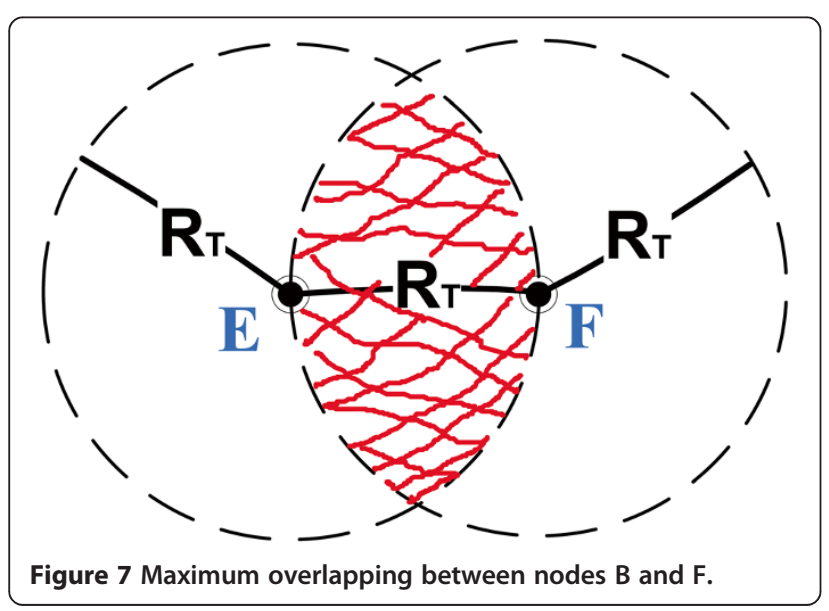

is necessary. To consider two nodes as two hop counts, the maximum overlapping between these two nodes is shown in Figure 7. The maximum overlapping area is $1.23 R_{\mathrm{T}}^{2}$. In a topology where nodes are uniformly distributed over the network area, the following condition guarantees the presence of at least $\eta$ nodes in the overlapping area [19]:

$$
N_{n} \geq \frac{\eta A_{N}}{1.23 R_{T}^{2}}
$$

To guarantee the overlapping of the transmission range of two nodes, the following requirement must be satisfied:

$$
N_{n}>\frac{\mathrm{A}_{N}}{R_{T}^{2}}
$$

This equation is in accordance with (28).

\subsubsection{PU activity dominates}

Figure $6 \mathrm{~b}$ shows the second scenario when the activity of the PU in the neighbourhood of node $\mathrm{E}$ may cause the links $A$ to $E$ or $E$ to $F$ to fail. This route failure occurs once 
the PU starts its transmission or when node E enters the coverage area of the PU.

\subsubsection{Spectrum heterogeneity and different channel transmission range}

The mobility of the CR user can also lead to spectrum handoff due to spectrum heterogeneity and various channel transmission ranges. Assume that two mobile nodes, with a distance less than $R_{l}$, are communicating in an active route, while they are using a channel of type $l$ for their data transmission. When their distance exceeds the $R_{l}$, they must change their communication channel and find a channel with a transmission range longer than $R_{l}$.

Therefore, as Figure 8 shows, the different handoff procedures are defined as:

- Forced intra-pool spectrum handoff: The operation frequency of the SU is changed to another spectrum band in the same spectrum pool. This type of handoff happens because of the appearance of the PU.

- Forced inter-pool spectrum handoff: The operation frequency of the SU is changed to another spectrum band in a different spectrum pool because of the appearance of the PU.

- Inter-pool spectrum handoff: The CR user changes its spectrum bands from one spectrum pool to another different spectrum pool. This type of spectrum handoff occurs because of the mobility and channel quality degradation of the SU.

- Local flow handoff: due to the SU mobility, there is no channel that can support the data transmission.

The spectrum handoff procedure happens when the current channel does not successfully send data. However, the spectrum handoff does not occur when the transmission

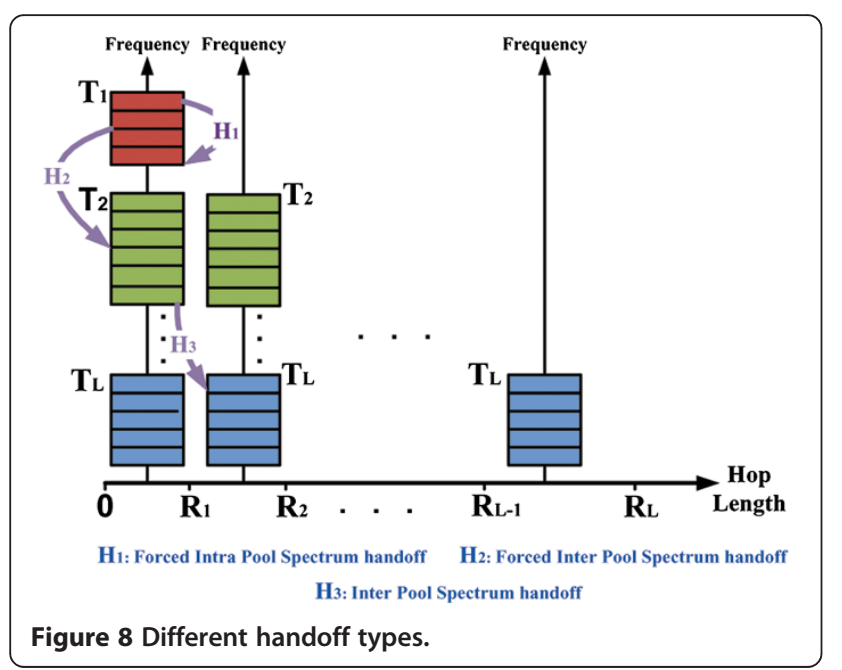

on any of all of the available channels fails. Therefore, the probability of spectrum handoff is equal to

$$
P_{\mathrm{SH}}=\sum_{i=1}^{L} \pi^{P}\left(T_{i}\right)\left[p_{\mathrm{uspt}, c_{i}}-\prod_{j=1}^{L} p_{\mathrm{uspt}, c_{j}}\right]
$$

In (30), the term $\sum_{i=1}^{L} \pi^{P}\left(T_{i}\right) \prod_{j=1}^{L} p_{\text {uspt }, c_{j}}$ is the probability that all available channels have unsuccessful packet transmission. In such a case, the troubled nodes do not perform spectrum handoff; they perform local flow handoff. Therefore, the probability of local flow handoff can be expressed as follows:

$$
P_{\mathrm{LH}}=\sum_{i=1}^{L} \pi^{P}\left(T_{i}\right) \prod_{j=1}^{L} p_{\mathrm{uspt}, c_{j}}
$$

The probability of successful spectrum handoff depends on the probability of successful packet transmission in a hop $p_{\text {spt }, c}$. However, to perform local flow handoff, Equations 28 and 29 must be satisfied. We define the link maintenance probability $\left(P_{\mathrm{LM}}\right)$ as the probability that the link is successfully maintained in a hop or between troubled nodes, which is dependent on the probability of channel availability between two nodes. Thus, the link maintenance probability, considering only spectrum handoff, can be written as follows:

$$
P_{\mathrm{LM}, \mathrm{SH}}=P_{\mathrm{SH}} P_{\mathrm{spt}, c}
$$

When the link maintenance is not successful, despite the spectrum handoff, local rerouting is performed. In this case, the probability of link maintenance is as follows:

$$
P_{\mathrm{LM}, \mathrm{LH}}=\left(\frac{N-2}{N}\right)\left(1-P_{\mathrm{LM}, \mathrm{SH}}\right) P_{\mathrm{LH}} P_{\mathrm{spt}, c}
$$

Finally, the probability of link maintenance, considering the unified routing and spectrum handoff management, can be stated as follows:

$$
P_{\mathrm{LM}, \mathrm{USH}}=P_{\mathrm{LM}, \mathrm{SH}}+P_{\mathrm{LM}, \mathrm{LH}}
$$

\section{Data rate of the secondary user}

For any secondary user that operates in a spectrum band, its maximal data rate can be represented by [31]

$$
D_{\mathrm{r}}=W_{c_{l}} \log _{2}\left(1+\frac{P_{\mathrm{T}} G_{c_{l}}}{n_{0}}\right)
$$

where $W_{c_{l}}$ is the bandwidth of the channel of type $l, n_{0}$ is the power of the additive white Gaussian noise (AWGN), $P_{\mathrm{T}}$ is the transmission power for SU and $G$ is the channel gain. Given the solutions of the steady state probabilities, we know that $\pi^{p}\left(T_{i}\right)$ is the stationary probability that 
the system is in state $T_{i}$, and thus, it can be thought of as the expected long-run fraction of the time that the Markov chain spends in the state $T_{i}$ [32]:

$$
\pi^{p}\left(T_{i}\right)=\lim _{\tau \rightarrow \infty} \frac{1}{\tau} \int_{0}^{\tau} \operatorname{Pr}\left\{T(t)=T_{i}\right\} d t
$$

where $T(t)$ is the state of the Markov chain at time $t$. Considering $D_{\mathrm{R}}$ as the long-run expected average throughput for the SU, it can be defined as follows:

$$
D_{\mathrm{R}}=\lim _{\tau \rightarrow \infty} \frac{1}{\tau} E\left(\int_{o}^{\tau} D_{\mathrm{r}}(T(t)) d t\right)
$$

where $D_{\mathrm{r}}(T(t))$ is the throughput of the SU achieved in state $T(t)$. Based on (35), (36) and (37), it can be concluded that

$$
D_{\mathrm{R}}=\lim _{\tau \rightarrow \infty} \frac{1}{\tau} E\left(\int_{o}^{\tau} D_{\mathrm{r}}(T(t)) d t\right)
$$

Therefore, by using the Markov chain, the dynamic utilization of the empty licensed bands for SUs without conflicting with the PU can be captured. Calculation of the spectrum utilization of the SUs from a statistical point of view and of their stationary behaviour is also possible.

\section{Results and discussion}

\subsection{Analytical results}

In the first part, analytical results are illustrated to support the analysis.

\section{- Channel availability and spectrum mobility consideration}

In this section, we show the effect of different parameters on the $P_{\mathrm{car}, c}$. In all of the following figures, we suppose that there are only two types of channels with a transmission range of $R_{1}=75 \mathrm{~m}$ and $R_{2}=125 \mathrm{~m}$ and a maximum node transmission range of $R_{T}=150 \mathrm{~m}$. We also assume that the activity of the PU on different channels is identical.

Figures 9 and 10 show the effect of type of the channels on the $P_{\text {car, } c}$. In these figures, $p=1 / 2, R_{1}=75 \mathrm{~m}$, $R_{2}=125 \mathrm{~m}, R_{T}=150 \mathrm{~m}, c=10$ and $N=8$. Figure 10 depicts the effect of the homogeneous channel transmission range on the probability of channel availability. The result implies that the $P_{\mathrm{car}, c}$ is dependent on the channel transmission range.

\section{- Link maintenance probability}

Spectrum heterogeneity and SU mobility have a significant effect on the handoff blocking probability in the integrated handoff management scheme. We define the probability of unsuccessful link maintenance as the probability of spectrum handoff blocking $\left(P_{\mathrm{hb}}\right)$. Figure 11 compares the probability of unsuccessful rerouting $\left(P_{\text {usrr }}\right)$, the handoff blocking probability deploying only spectrum handoff $\left(P_{\mathrm{hb}, \mathrm{SH}}\right)$ and the handoff blocking probability deploying integrated handoff management $\left(P_{\mathrm{hb}, \mathrm{USH}}\right)$, considering various number of $\mathrm{SU}$ nodes in the network. In the following figures, $L=2, p=.5, R_{1}=75 \mathrm{~m}$, $R_{2}=125 \mathrm{~m}, R_{T}=150, c=10, c_{1}=5, c_{2}=5$ and the number of hops is equal to 14 . Based on this figure, the integrated handoff management scheme outperforms the scheme deploying only spectrum handoff in terms of link maintenance probability. The probability of link maintenance in the integrated handoff scheme is also significantly higher than the probability of successful rerouting. As the number of SUs in the network increases, the probability of handoff blocking decreases because the

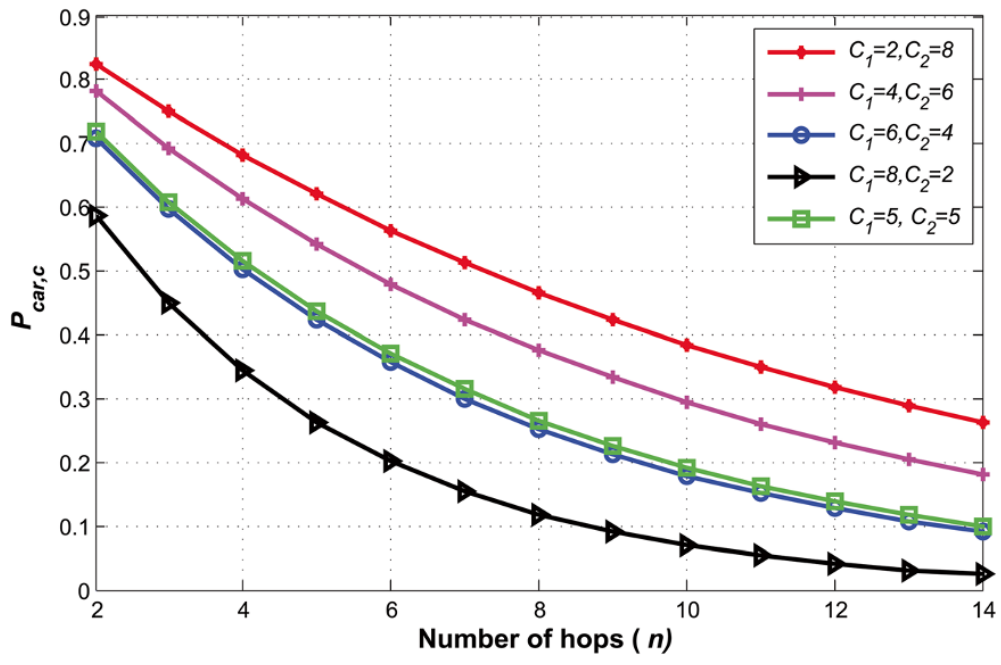

Figure 9 The effect of the number of different available channels on $P_{\mathrm{car}, c}$. 


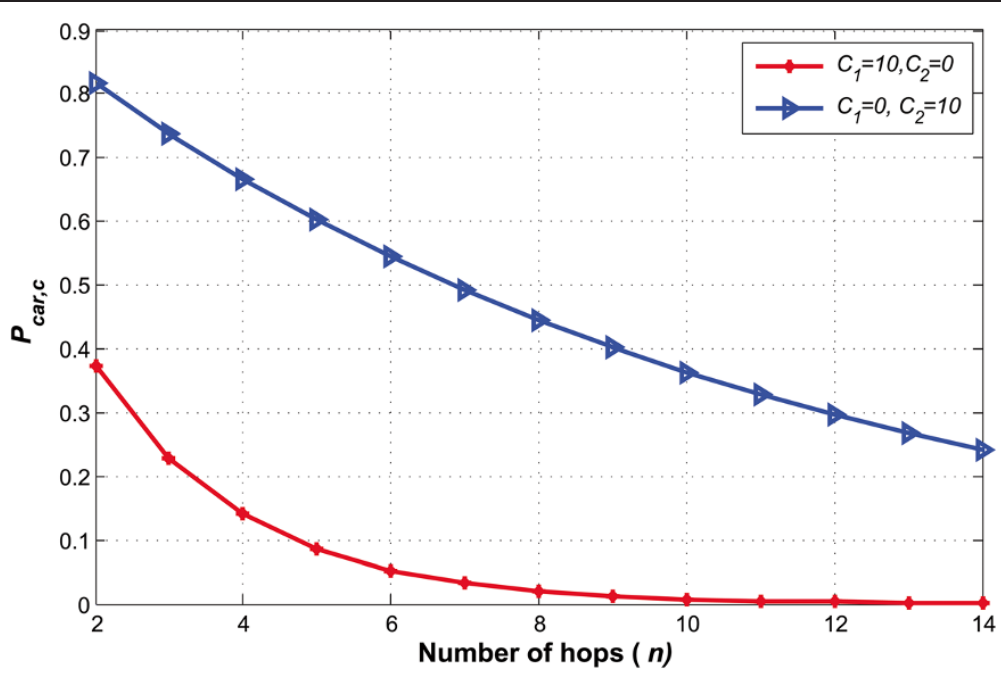

Figure 10 The effect of the channel transmission range on $P_{c a r, c}$.

probability of finding the proper nodes, which work as the relay, to perform local rerouting increases.

Based on [33], with an area network of $\mathrm{AN}=1,000 \mathrm{~m}^{2}$ and $R_{\mathrm{T}}=150 \mathrm{~m}$, the number of expected hop counts in the network is equal to five. Figure 12 compares the proposed parameters in Figure 11 with an expected hop count in the network equal to five. Comparing Figures 11 and 12, we conclude that the probability of handoff blocking decreases when the number of hops decreases. This study shows that the proposed integrated spectrum handoff management and routing scheme achieves more actual data transmission opportunities.

- SU's data rate
Figure 13 compares the effects of channel heterogeneity and PU activity on the SU data rate. Based on this figure, the higher transmission range leads to a higher data rate because the number of handoffs will be reduced. Therefore, the effect of channel heterogeneity in terms of transmission range and path loss must be considered on the performance of the integrated handoff management.

\subsection{Simulation results}

In this subsection, performance comparisons of three different schemes are conducted using Network Simulator 2 (ns-2) [34]. To study the handoff blocking probability, three different handoff management schemes are considered. These three different versions of the handoff management scheme are defined as the SH scheme,

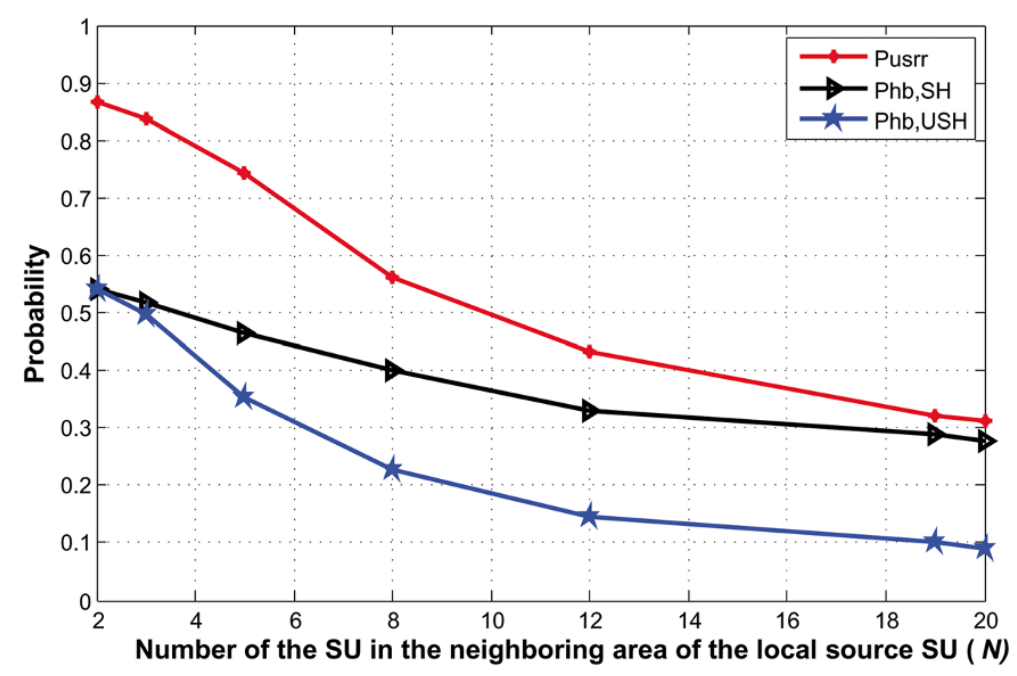

Figure 11 Comparison of different handoff management schemes and routing performance with a hop count of 14 . 


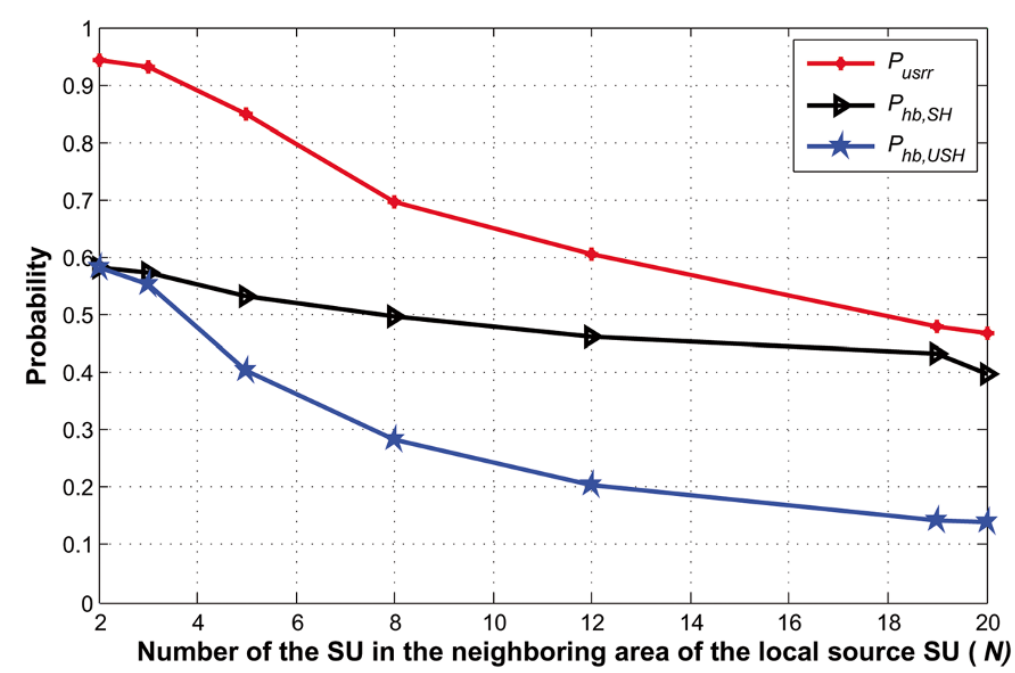

Figure 12 Comparison of different handoff management schemes and routing performance with an expected hop count of five.

the reactive unified spectrum handoff (USH) scheme and the proactive unified spectrum handoff (PUSH) scheme. The first scheme only deploys the spectrum handoff, while the remaining two schemes deploy the unified spectrum handoff in which the local flow handoff will be added to the management system. One of these two schemes, USH, does not consider the handoff threshold; whereas PUSH considers the handoff threshold for the preemptive handoff region.

There is a total number of available channels $C=10$, classified into two different types $C_{1}=5$ and $C_{2}=5$. The transmission ranges of different channel types are set to $R_{1}=75 \mathrm{~m}$ and $R_{2}=125 \mathrm{~m}$. The mobile SUs are distributed in a network with a 2,000 $\mathrm{m} \times 2,000 \mathrm{~m}$ area, and their speed is set to $3 \mathrm{~m} / \mathrm{s}$. The transmission range of the static PUs is set to $200 \mathrm{~m}$, and the activity of PUs is modelled as a two-stage on/off procedure with an exponential distribution.

Here, two different handoff thresholds are defined. The signal power of hello packets is used to approximate the distance between the transmitter and the receiver. Suppose that $P_{\text {snd }}$ is the hello packet signal power at the transmitting antenna and $P_{\mathrm{r}}$ is the receiving power at distance $r$. Based on [35], the signal power received through free space decreases with distance such that:

$$
P_{\mathrm{r}}=\frac{P_{\text {snd }}}{r^{n}}
$$

where $n$ is a number typically between 2 and 4 (e.g. equal to 2 near the transmitter until a certain point at which $n$ becomes 4) [35]. Because the two preemptive regions are near the maximum transmission range, we

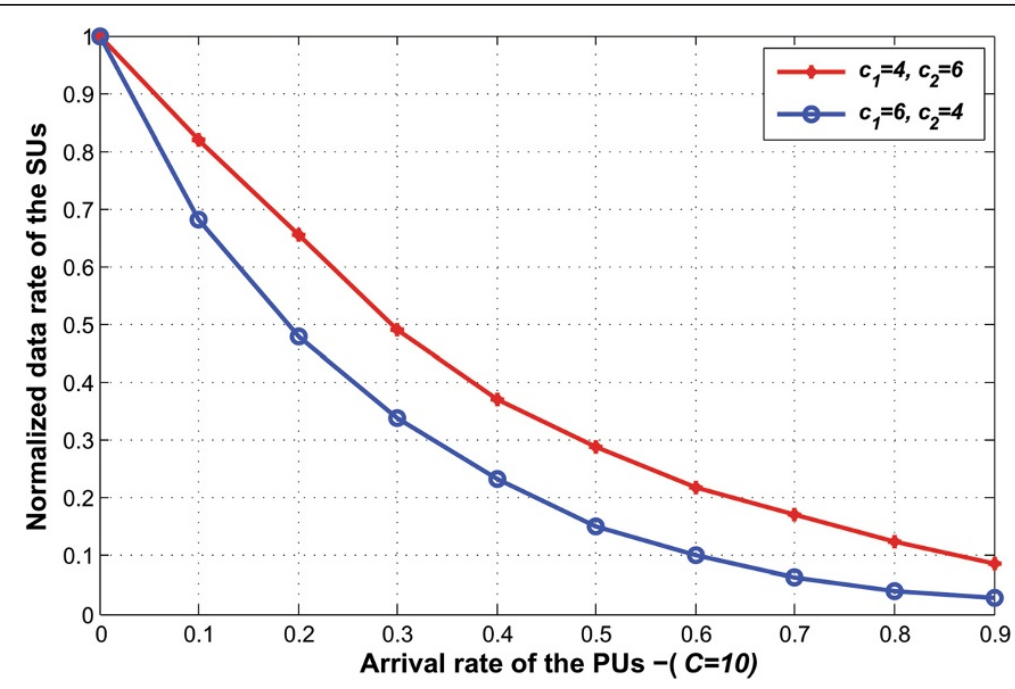

Figure 13 Effects of the channel heterogeneity and PU activity on the SU data rate. 


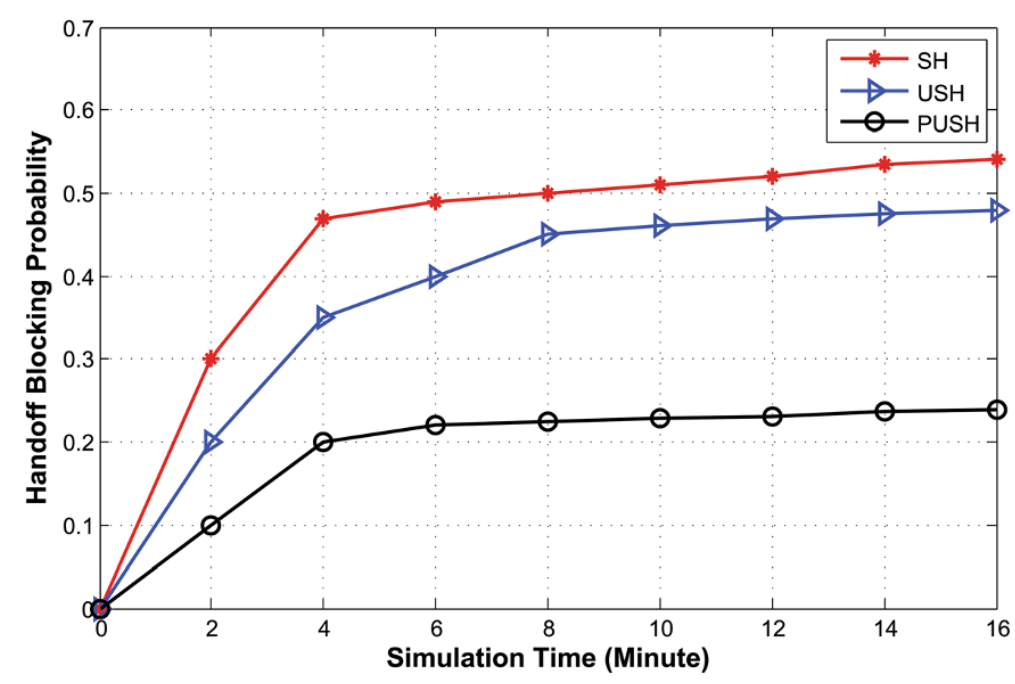

Figure 14 Comparison of the performance of different handoff management schemes in terms of handoff blocking probability.

model the drop in the signal power to $1 / r^{4}$ throughout these regions:

$$
P_{\mathrm{r}}=\frac{P_{\text {snd }}}{r^{4}}
$$

Based on (34), the signal power threshold for preemptive channel handoff $\left(P_{\mathrm{STPCH}}\right)$ can be expressed as follows:

$$
\begin{aligned}
P_{\mathrm{STPCH}, l} & =\frac{P_{\text {snd }}}{\left(R_{l}-W_{\mathrm{ch}, l}\right)^{4}} \\
& =\frac{P_{\text {snd }}}{\left(R_{l}-\left(v_{\text {relative }} \times t_{\mathrm{w}, l}\right)\right)^{4}}
\end{aligned}
$$

where the $W_{\mathrm{ch}, l}$ is the warning distance for nodes communicating on a channel of type $l, v_{\text {relative }}$ is the relative speed of nodes, and $t_{\mathrm{w}, l}$ is the warning interval. The minimum power received by the receiver, which is the power at the channel transmission range, $P_{\mathrm{CTR}, l}$, is expressed as follows:

$$
P_{\mathrm{CTR}, l}=\frac{P_{\mathrm{snd}}}{R_{l}{ }^{4}}
$$

The spectrum handoff threshold (SHTH) can be described as:

$$
\mathrm{SHTH}, l=\frac{P_{\mathrm{STPCH}, l}}{P_{\mathrm{CTR}, l}}=\frac{R_{l}{ }^{4}}{\left(R_{l}-\left(v_{\text {relative }} \times t_{\mathrm{w}, l}\right)\right)^{4}}
$$

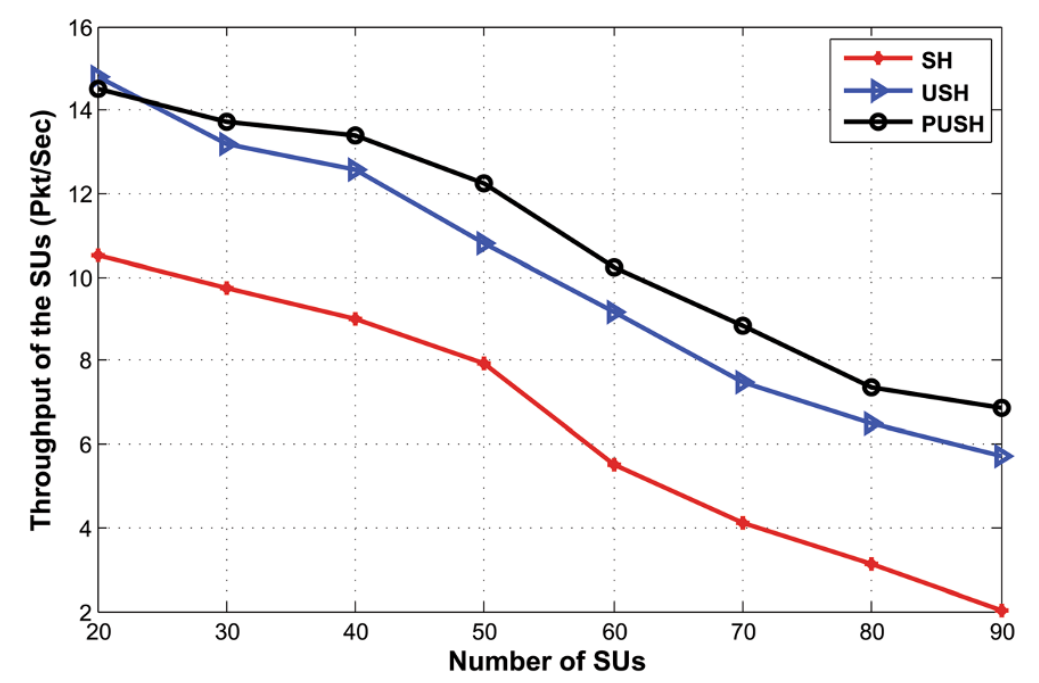

Figure 15 Comparison of performance of different handoff management schemes of SU's throughput vs. number of SUs. 


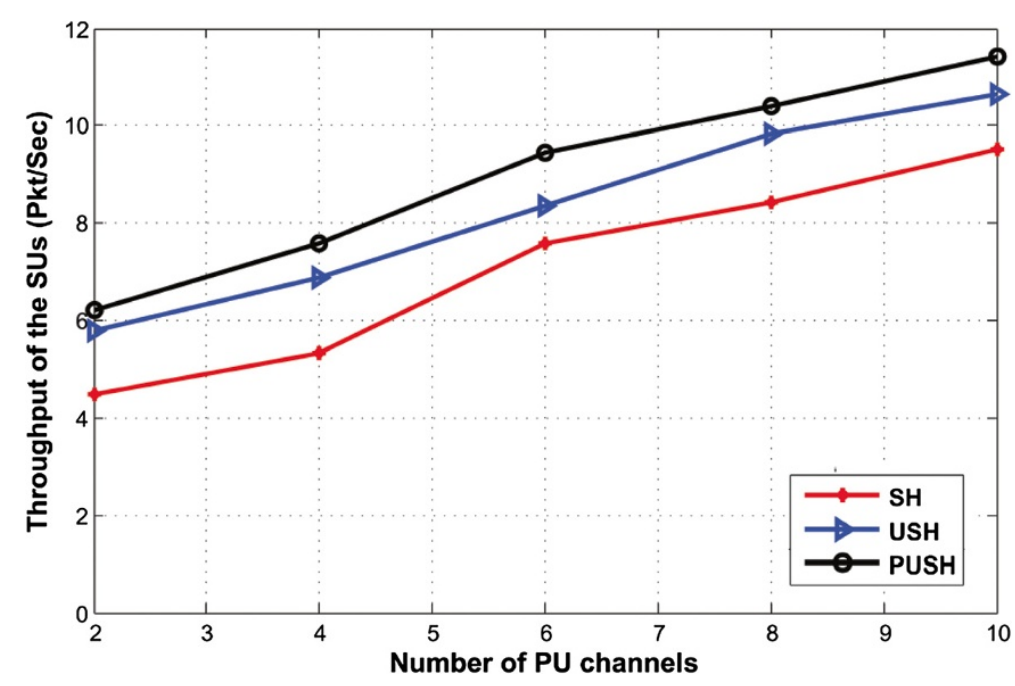

Figure 16 Comparison of performance of different management schemes of SU's throughput vs. number of PU channels.

Similarly, the handoff threshold for the preemptive local flow handoff region (LHTH) can be expressed as:

$$
\mathrm{LHTH}=\frac{P_{\mathrm{SLFTH}}}{P_{\mathrm{NTR}}}=\frac{R_{T}{ }^{4}}{\left(R_{T}-W_{\text {link }}\right)^{4}}
$$

where $P_{\text {SLFTH }}$ is the signal power threshold for preemptive flow handoff and $P_{\mathrm{NTR}}$ indicates the minimum power received by the receiver at the node transmission range, $R_{T}$. The $t_{\mathrm{w}, l}$, which is the interval from the warning till the communication link break off, needs to be greater than or equal to the necessary time for performing the handoff.

- Link maintenance, handoff blocking, handoff delay and spectrum handoff performance
In this part, the SU's route maintenance probability or handoff blocking probability is investigated. The handoff threshold time is set to $6 \mathrm{~s}$, and both the PU's and the SU's arrival rates are set to be 0.25 . The reactive AODV [36] routing protocol is used for route formation over CR-MANET.

Figure 14 verifies the effect of local flow handoff on the route maintenance probability. It is expressed that the proposed unified handoff approach efficiently improves the route maintenance probability. In this approach, the data flow is transferred to the nearby users to keep the communication while the first scheme only uses the spectrum handoff to keep the route. The probability of unsuccessful route maintenance is defined as the probability of spectrum handoff blocking $\left(P_{\mathrm{hb}}\right)$. Figure 14 indicates that the spectrum handoff blocking probability in the PUSH

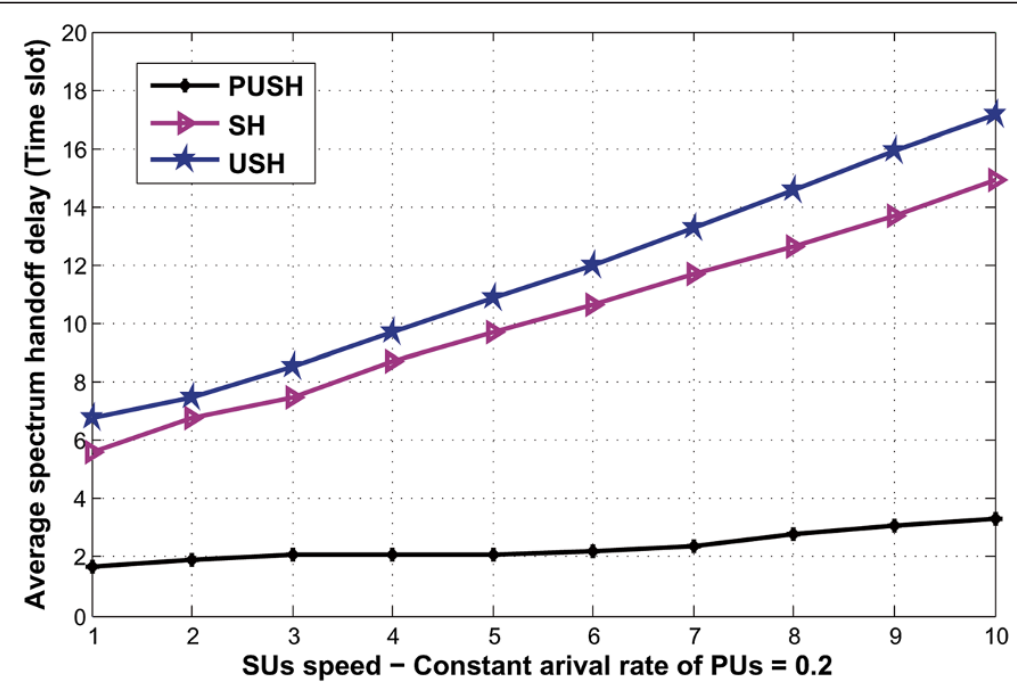

Figure 17 Average spectrum handoff delay for the SUs versus the velocity of SUs. 


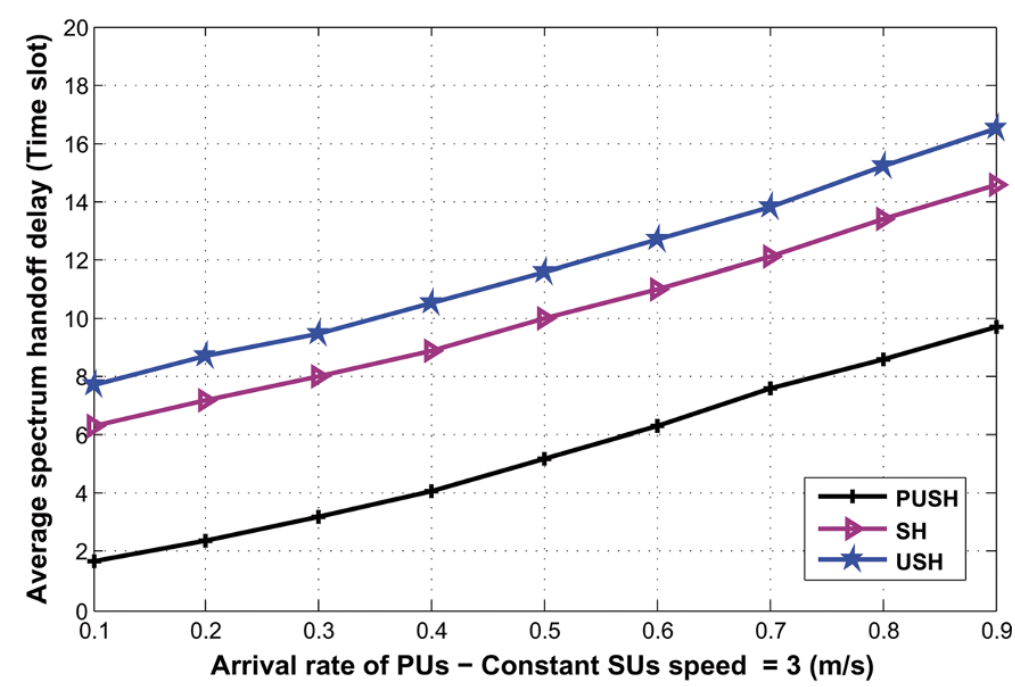

Figure 18 Average spectrum handoff delay under different arrival rates of PUs.

scheme $\left(P_{\mathrm{hb}, \mathrm{PUSH}}\right)$ is significantly lower than the probability of the spectrum handoff blocking in the scheme deploying only spectrum handoff $\left(\mathrm{P}_{\mathrm{hb}, \mathrm{SH}}\right)$. This is due to deploying the local flow handoff in PUSH. On the other hand, the $P_{\mathrm{hb}, \mathrm{PUSH}}$ is lower than the probability of handoff blocking in the scheme deploying reactive unified spectrum handoff $\left(\mathrm{P}_{\mathrm{hb}, \mathrm{USH}}\right)$. This is because PUSH performs the handoff proactively. Hence, deploying the proactive local flow handoff can be more efficient than the other schemes.

Figure 15 shows the SU throughput with various numbers of SUs. In this part, the arrival rate of SU packets is equal to 200 packets per second. The PU's packet has an arrival rate equal to 10 packets per second. It is significant that the throughput of SU transmissions decreases when the number of SUs increases. This is because more SUs result in fewer opportunities to capture the channel for each SU. In this figure, on the other hand, the PUSH scheme outperforms the other two schemes in terms of SU throughput.

Figure 16 shows the $\mathrm{SU}$ throughput under various numbers of PU channels. In this part, the arrival rate of SU and PU packets are the same as in Figure 15. The number of PU channels varies and is divided into identical numbers of channels belonging to two different types detectable by any SU at any location. The number of SUs in the network is fixed at 50 . As the number of PU channels increases, the SUs' throughput increases because more channels can be captured by the SUs for data transmissions. In this figure, the PUSH scheme outperforms the two other schemes in terms of SU throughput.

Figures 17 and 18 show the simulation results of the average spectrum handoff delay under different network

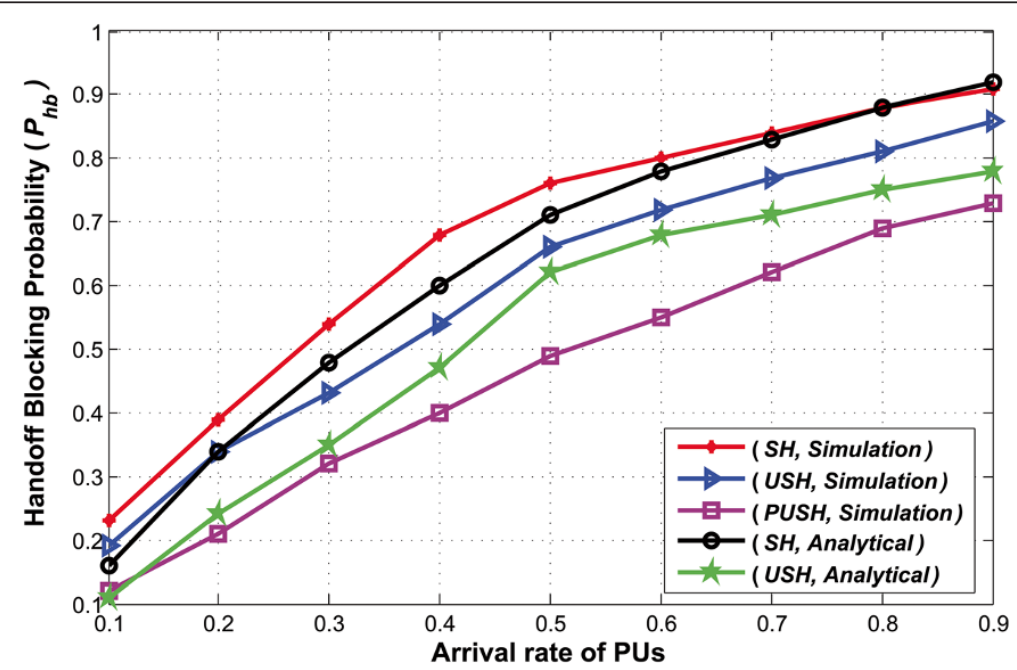

Figure 19 Comparison of different handoff management schemes performance in terms of handoff blocking probability. 
conditions. From these figures, it can be stated that by using the integrated handoff management scheme, the average spectrum handoff delay is reduced significantly.

Figure 19 compares the handoff blocking probability in analytical model considering various arrival rates of the PUs in the network. This figure also compares three different handoff management schemes to study the handoff blocking probability based on the simulation results. Based on this figure, in the analytical model, the unified handoff management scheme outperforms the scheme deploying only spectrum handoff in terms of link maintenance probability, which is also verified by the simulation results. The simulation results indicate that the spectrum handoff blocking probability in the PUSH scheme $\left(P_{\mathrm{hb}, \mathrm{PUSH}}\right)$ is significantly lower than the probability of the spectrum handoff blocking in the scheme deploying only spectrum handoff $\left(\mathrm{P}_{\mathrm{hb}}, \mathrm{SH}\right)$. This is due to deploying the local flow handoff (LFH) in PUSH. On the other hand, the $P_{\mathrm{hb}, \mathrm{PUSH}}$ is lower than the probability of handoff blocking in the scheme deploying USH $\left(\mathrm{P}_{\mathrm{hb}, \mathrm{USH}}\right)$. This is because the PUSH performs the handoff proactively. Hence, deploying the proactive LFH can be more efficient than the other schemes.

\section{Conclusions}

Spectrum handoff management is still an open issue in CR networks. It is particularly challenging in CR-MANETs. In CR-MANETs, the available spectrum bands vary over time and space, while they are distributed nonadjacently over a broad frequency range. However, in CR-MANETs, the fluctuation of PU activity and the SU mobility make the issue of maintaining optimal routes more complex. In this work, we present an integrated spectrum handoff management and routing scheme that considers spectrum mobility in the time and space domains and considers the network topology variations. We propose a network architecture that considers the heterogeneous spectrum availability and its variation over time and space and distributed nodes. Then, the probability of channel availability in this dynamic radio environment is calculated. Based on this unified architecture, an integrated routing and spectrum handoff management scheme is proposed. The proposed scheme considers the CR-MANETs spectrum handoff problem and incorporates the routing issue. Both the analytical and simulation results verify the improvement in the network performance using the introduced management scheme.

\section{Competing interests}

The authors declare that they have no competing interests.

\section{Authors' information}

$\mathrm{SN}$ is a researcher of the Universiti Teknologi Malaysia under the Post Doctoral Fellowship Scheme for the Project: 'SELF ORGANIZING INTELLIGENT-BASED COGNITIVE RADIO LEARNING SYSTEM FOR INTELLIGENT P-PUSH'.

\section{Acknowledgements}

This work was supported in part by the Ministry of Science, Technology and Innovation (MOSTI) Malaysia, and the Research Management Center (RMC), University Technology Malaysia under GUP research grant no. R. J130000.7923.4S063.

Received: 25 March 2014 Accepted: 26 August 2014

Published: 7 September 2014

\section{References}

1. V Chakravarthy, L Xue, W Zhiqiang, A Michael, F Garber, R Kannan, A Vasilakos, Novel overlay/underlay cognitive radio waveforms using SD-SMSE framework to enhance spectrum efficiency- part i: theoretical framework and analysis in AWGN channel. IEEE Trans. Commun. 57(12), 3794-3804 (2009)

2. J Mitola, GQ Maguire, Cognitive radio: making software radios more personal. Pers. Commun. IEEE 6(4), 13-18 (1999)

3. A Attar, H Tang, A Vasilakos, F Yu, V Leung, A Survey of security challenges in cognitive radio networks: solutions and future research directions. Proc. IEEE 100(12), 3172-3186 (2012)

4. AW Min, KG Shin, Impact of mobility on spectrum sensing in cognitive radio networks, in Proceedings of the 2009 ACM Workshop on Cognitive Radio Networks CoRoNet (Beijing, China, 2009), p. 13

5. WY Lee, Spectrum management in cognitive radio wireless networks, in Thesis, (PHD) Georgia Institute of Technology, 2009

6. IF Akyildiz, WY Lee, KR Chowdhury, CRAHNs: cognitive radio ad hoc networks. Ad Hoc Netw. 7, 810-836 (2009)

7. X Huang, D Lu, $\mathrm{P} L \mathrm{Li}, \mathrm{Y}$ Fang, Coolest path: spectrum mobility aware routing metrics in Cognitive Ad Hoc Networks, in Distributed Computing Systems (ICDCS), 31st International Conference on (Minneapolis, 2011), pp. 182-191

8. B Sang-Seon, I Balashingham, A Vasilakos, L Heung-No, Computation of an equilibrium in spectrum markets for cognitive radio networks. IEEE Trans. Comput. 63(2), 304-316 (2014)

9. B Sang-Seon, B llangko, V Vasilakos, A market-clearing model for spectrum trade in cognitive radio networks, in Proceedings of the Twelfth ACM International Symposium on Mobile Ad Hoc Networking and Computing (MobiHoc'11) (ACM, New York, 2011)

10. M Youssef, M Ibrahim, M Abdelatif, C Lin, A Vasilakos, Routing metrics of cognitive radio networks: a survey. Commun. Surv. Tut. IEEE 16(1), 92-109 (2014)

11. W Magbool, SK Syed-Yusof, NM Abdul-Latiff, S Nejatian, Characterization of end-to-end path selection for cognitive radio wireless mesh networks. Life Sci. J. 11(8), 827-834 (2014)

12. L Giupponi, Al Perez-Neira, Fuzzy-based spectrum handoff in cognitive radio networks, in 3rd International Conference on Cognitive Radio Oriented Wireless Networks and Communications CrownCom (Singapore, 2008), pp. 1-6

13. W Feng, J Cao, C Zhang, C Liu, Joint optimization of spectrum handoff scheduling and routing in multi-hop multi-radio cognitive networks, in 29th IEEE International Conference on Distributed Computing Systems (Montreal, 2009), pp. 85-92

14. Y Song, J Xie, ProSpect: a proactive spectrum handoff framework for cognitive radio ad hoc networks without common control channel. IEEE Trans. Mobile Comp. 11(7), 1127-1139 (2012)

15. Y Song, J Xie, Performance analysis of spectrum handoff for cognitive radio ad hoc networks without common control channel under homogeneous primary traffic, in INFOCOM, 2011 Proceedings IEEE (Shanghai, 2011), pp. 3011-3019

16. Z Damljanovic, Mobility Management Strategies in Heterogeneous Cognitive Radio Networks. J. Netw. Syst. Manage 18(1), 4-22 (2010)

17. J Duan, $Y \mathrm{LI}$, An optimal spectrum handoff scheme for cognitive radio mobile ad hoc networks. Digit Object Identifier 11, 11-16 (2011)

18. $\mathrm{KWu}, \mathrm{J}$ Harms, Performance study of proactive flow handoff for mobile ad hoc networks. Wirel. Netw 12, 119-135 (2006)

19. P Abhilash, S Perur, S lyer, Router handoff: an approach for preemptive route repair in mobile ad hoc networks. Lect. Notes Comp. Sci. 2552, 347-357 (2002)

20. J Tigang, W Honggang, A Vasilakos, QoE-driven channel allocation schemes for multimedia transmission of priority-based secondary users over cognitive radio networks. IEEE J. Selected Areas Commun. 30(7), 1215-1224 (2012)

21. A Chehata, An on-demand routing protocol for multi-hop multi-radio multi-channel cognitive radio networks, in Wireless Days 2011 IEEE Conferences (Niagara Falls, 2011), pp. 1-5 
22. AS Cacciapuoti, M Caleffi, L Paura, Reactive routing for mobile cognitive radio ad hoc networks. Ad Hoc Netw. 10(5), 803-815 (2012)

23. A Cianfrani, V Eramo, M Listanti, M Polverini, A Vasilakos, An OSPF-integrated routing strategy for QoS-aware energy saving in IP backbone networks. IEEE Trans. Netw. Serv. Manage 9(3), 254-267 (2012)

24. S Nejatian, SK Syed-Yusof, NM Abdul Lattif, V Asadpour, Proactive integrated handoff management in CR-MANETs: a conceptual model, in IEEE Symposium on Wireless Technology and Applications (ISWTA) (Bandung, 2012), pp. 33-38

25. S Nejatian, JA Abolarinwa, SK Syed-Yusof, NM Abdul Lattif, V Asadpour, Characterization of spectrum mobility and channel availability in CR-MANETs, in Proc. of the International Conference on Advances in Mobile Networks and Communication - MNC 2012 (Kuala Lumpur, 2012), pp. 12-16

26. S Nejatian, SK Syed-Yusof, NM Abdul Lattif, N Fisal, V Asadpour, Handoff management in cognitive radio networks: Concepts, protocols, metrics and challenges. Int. Rev. Comput. Softw. (I.RE.CO.S.) 7(6), 2993-3006 (2012)

27. S Nejatian, SK Syed-Yusof, NM Abdul Lattif, V Asadpour, H Hosseini, Proactive integrated handoff management in cognitive radio mobile ad hoc networks. EURASIP J. Wireless Commun. Netw. 224, (2013). doi:10.1186/1687-1499-2013224

28. S Nejatian, SK Syed-Yusof, NM Abdul-Latiff, V Asadpour, IEEE conference on Personal Indoor and Mobile Radio Communications (PIMRC), (IEEE 24th International Symposium on, 2013). ISSN 2166-9570, 28872892

29. T-C Hou, VO-K Li, Transmission range control in multihop packet radio networks. IEEE Trans. Commun. 32(1), 38-44 (1986)

30. L Peng, G Song, Y Shui, A Vasilakos, CodePipe: an opportunistic feeding and routing protocol for reliable multicast with pipelined network coding, in INFOCOM, 2012 Proceedings IEEE (Orlando, 2012), pp. 100-108

31. TM Cover, JA Thomas, Elements of Information Theory (Wiley-Interscience, NewYork, 1990)

32. VG Kulkarni, Modeling and Analysis of Stochastic Systems (CRC, Boca Raton, 1995)

33. J Li, C Blake, DSJ De Couto, HI Lee, R Morris, Capacity of ad hoc wireless networks. Network 01, 61-69 (2001)

34. The NS Manual (formerly ns Notes and documentation) (Vint Project, Berkeley, CA, USA, 2010)

35. T Goff, NB Abu-Ghazleh, DS Phatak, R Kahvecioglu, Preemptive routing in ad hoc networks. J. Parallel Distributed Comput. 63(2), 123-140 (2003)

36. CE Perkins, EM Belding-Royer, Ad hoc on demand distance vector (AODV), in Proc. Of IEEE WMCSA (New Orleans, 1999), pp. 90-100

doi:10.1186/1687-1499-2014-144

Cite this article as: Nejatian et al:: Modelling of routing and spectrum handoff in CR-MANETs. EURASIP Journal on Wireless Communications and Networking 2014 2014:144.

\section{Submit your manuscript to a SpringerOpen ${ }^{\circ}$ journal and benefit from:}

- Convenient online submission

- Rigorous peer review

- Immediate publication on acceptance

- Open access: articles freely available online

- High visibility within the field

- Retaining the copyright to your article

Submit your next manuscript at $\gg$ springeropen.com 